\title{
Cell communication by periodic cyclic-AMP pulses
}

\author{
By G. Gerisch, D. Hülser, D. Malghow and U. Wigk \\ Friedrich-Miescher-Laboratorium der Max-Planck-Gesellschaft and \\ Max-Planck-Institut für Virusforschung, 74 Tübingen, Germany
}

[Plate 3]

At the surface of aggregating cells of the slime mould, Dictyostelium discoideum, two different sites interacting with extracellular cAMP are detectable: binding sites and cyclic-nucleotide phosphodiesterase. Both sites are developmentally regulated. An adequate stimulus for the chemoreceptor system in $D$. discoideum is the change of cAMP concentration in time, rather than concentration per se: long-term binding of cAMP causes only a short-term response. The system is, consequently, adapted to the recognition of pulses rather than to steady-state concentrations of cAMP. The cells are, nevertheless, able to sense stationary spatial gradients and to respond to them by chemotactic orientation. The possibility is discussed that they do so by transforming spatial concentration changes into temporal ones, using extending pseudopods as sensors.

The cAMP recognition system is part of a molecular network involved in the generation of spatio-temporal patterns of cellular activities. This system controls the periodic formation of chemotactic signals and their propagation from cell to cell. The phosphodiesterase limits the duration of the cAMP pulses and thus sharply separates the periods of signalling; the binding sites at the cell surface are supposed to be the chemoreceptors.

The control of cellular activities via cAMP receptors can be studied with biochemical techniques with cell suspensions in which spatial inhomogeneities are suppressed by intense stirring, whereas the temporal aspect of the spatiotemporal pattern is preserved. Under these conditions it can be shown that the extracellular cAMP concentration changes periodically, and that the phase of the cellular oscillator can be shifted by external pulses of cAMP. It can also be shown that small cAMP pulses induce a high output of cAMP, which demonstrates signal amplification, a function necessary for a cellular relay system.

\section{Periodic signalling, signal relaying and chemotactic response: mechanisms of cell aggregation in the slime mould, Dictyostelium discoideum}

Cell interactions in animal development result in the formation of spatio-temporal patterns of cellular activities. Their consequence is the organization of an embryo with its precisely ordered spatial arrangement of differentiated cells. In this paper we discuss a primitive system of morphogenetic cell interactions also ordered in space and time, which because of its simplicity is amenable to an analysis of signalling and signal recognition at the molecular level, and which might function as a model for the complicated systems as they have to be expected during embryogenesis of higher animals and man.

Cell aggregation in the slime mould, Dictyostelium discoideum, is a developmental step which links the single-cell state in which this organism exists as a population of independently growing amoebae, to a multicellular state. Later on, development culminates into the formation of a fruiting body which consists of two types of differentiated cells: spores and cells forming a stalk. During aggregation the cells are guided by diffusible transmitter(s) to aggregation centres where the multicellular body is formed. The signal system which mediates cell communication 
via extracellular transmitter can be separated into the following more elementary functions: (1) chemotaxis, the orientated cell movement in concentration gradients of an attractant, (2) relaying of the signals from cell to cell and (3) periodic generation of the signals from established centres (figure 1). Spatiotemporal patterns of these cellular activities arise within an hour by 'self-organization' from uniform fields of cells (4). In cell layers these activity patterns have the two-dimensional shape of spirals or concentric waves that spread from the centres to the boundaries of aggregation territories, where, in the typical case, they extinguish each other. This behaviour indicates that signalling is followed by a period of relative refractoriness (Shaffer 1962; Gerisch 1968, 1971; Cohen \& Robertson 1971; Durston 1974).
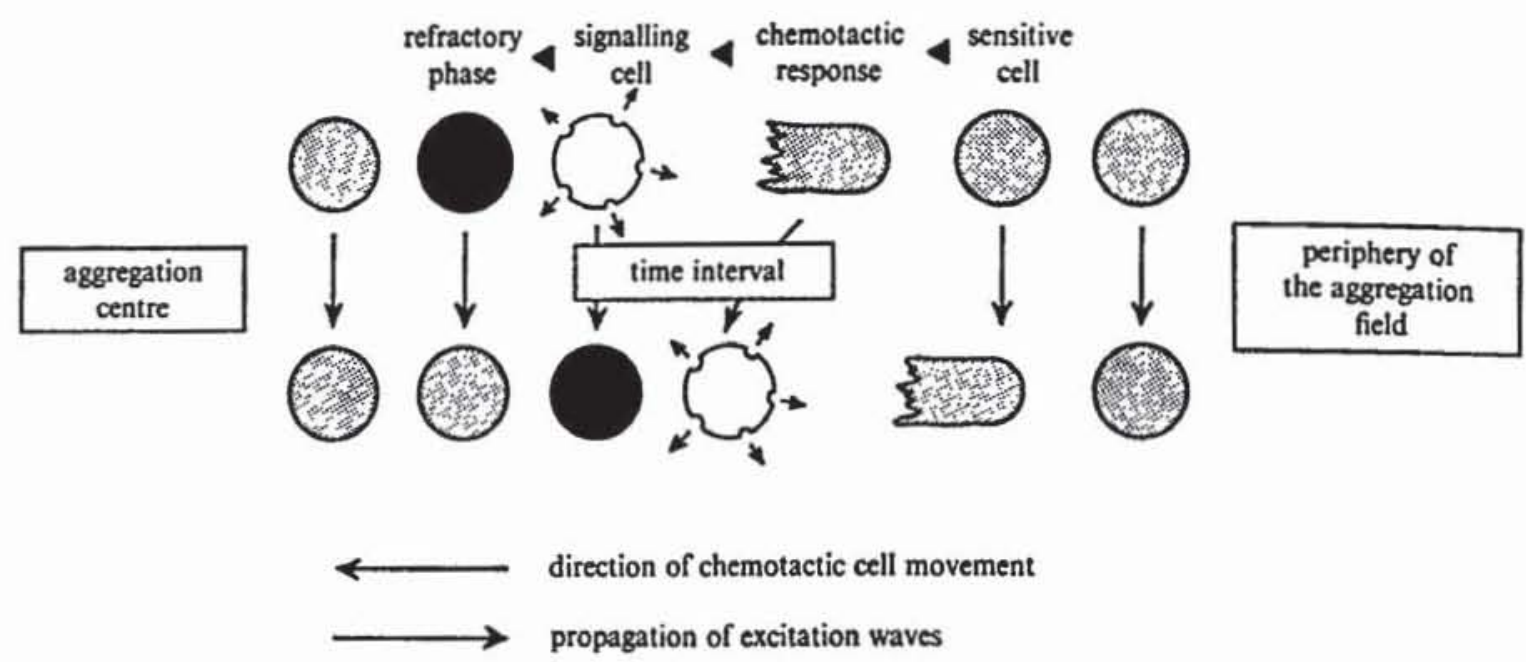

Figure 1. Simplified diagram of the communication of aggregating $D$. discoideum cells by diffusible transmitter. Periodic pulses originate in the aggregation centre (left) and are relayed in a cell layer. Signalling cells stimulate others to respond chemotactically and, after a time interval, to release their own transmitter, thus continuing the signal chain. In each period, a cell undergoes a cycle in the sequence from the right to the left as indicated on top. The refractory phase accounts for unidirectional signal propagation and for absence of wave reflexion at the boundaries of the territory. Refractoriness refers to a state after signalling in which the ability of a cell to relay a signal is reduced but not necessarily zero. It is an open question if the asymmetry of the chemotactic response (only from right to left) is due to a second chemical signal, not identical with cAMP, which would inhibit chemotactic orientation in the phase after signalling (Gerisch, Normann \& Beug 1966).

Cyclic AMP has been shown to be an intercellular signal for aggregating $D$. discoideum cells, and attempts have been made to interpret the observed phenomena by assuming that cAMP has multiple functions in cell communication, acting as a chemotactic factor as well as a transmitter for propagated signals (Konijn 1972; Bonner et al. 1969; Robertson, Drage \& Cohen 1972). In this context, the properties of the cAMP receptor system and the functional connexion of the receptors to the processes underlying chemotaxis, periodic pulse generation and signal relaying are of fundamental importance for the generation of the dynamical patterns of cellular activities observed at the multicellular level.

\section{Cyclic-AMP recognition: binding sites and cell-surface phosphodiesterase}

The first cell surface site we found to interact with extracellular cAMP is a phosphodiesterase which has a high activity in aggregating cells, and a low activity in growth-phase cells (Pannbacker \& Bravard 1972; Malchow, Nägele, Schwarz \& Gerisch 1972). These results prompted a study on the relation of the enzyme to the cAMP receptor mediating the chemotactic response (figure 2). Among a series of cAMP analogues and other cyclic nucleotides tested, most 


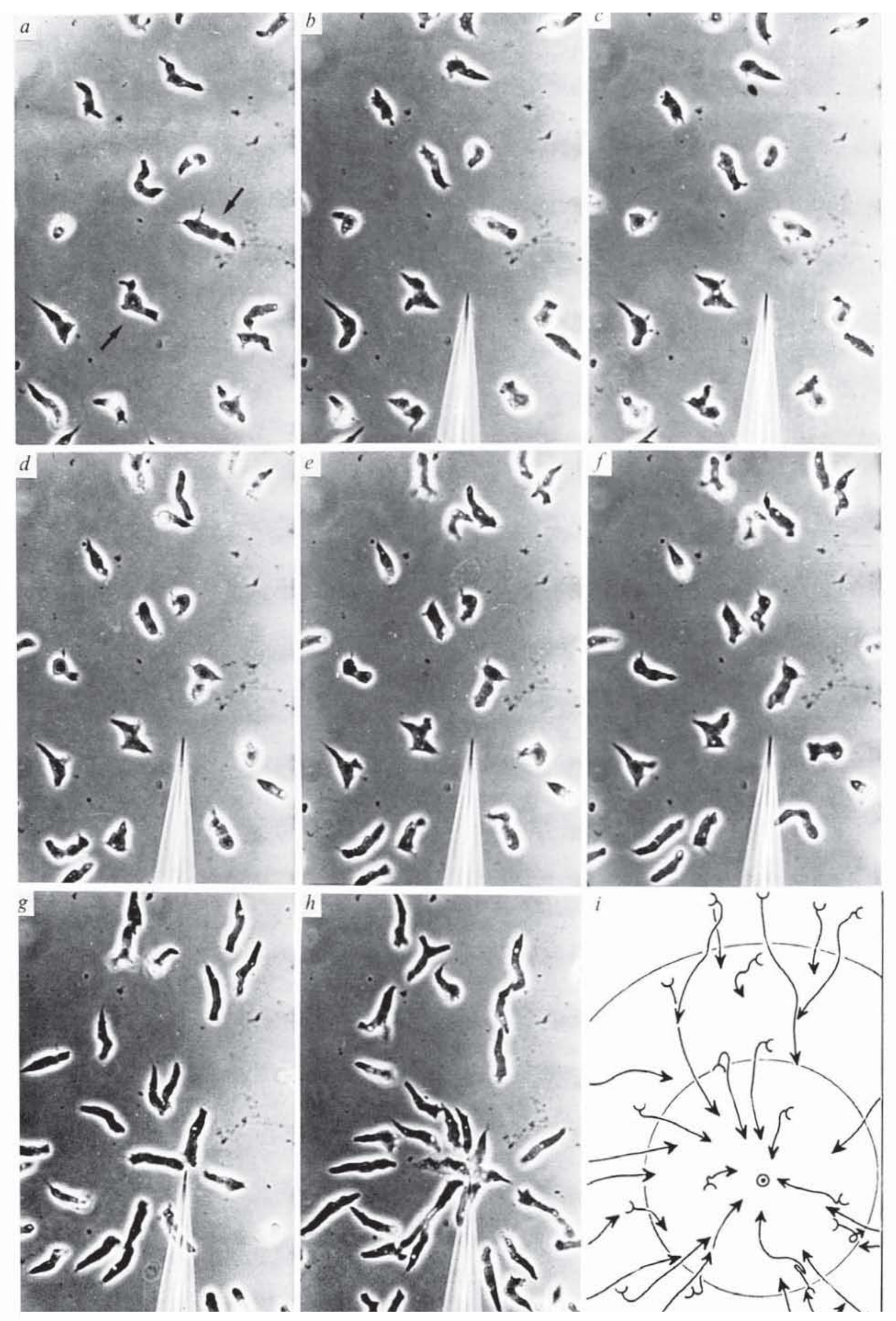

Figure 3. For description sce opposite. 
showed an affinity to the enzyme comparable to their chemotactic activity. Cyclic GMP, however, was an exception: it blocked the enzyme (Malchow, Fuchila \& Jastorff 1973) but had only a low chemotactic activity (Konijn 1972), indicating weak interaction with the cAMP receptor. Consequently, we used cyclic GMP to preferentially block the enzyme. The delay of cAMP hydrolysis caused by cGMP resulted in the discovery of other cAMP binding sites which, like the enzyme, are developmentally regulated cell surface components and fulfil the requirements of cAMP receptors in all respects hitherto tested (Malchow \& Gerisch 1973,

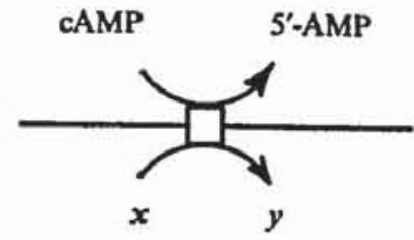

(1)

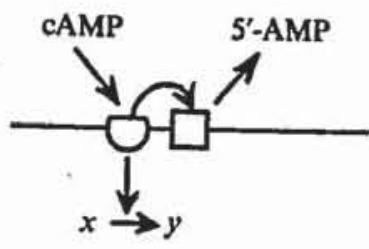

(2)

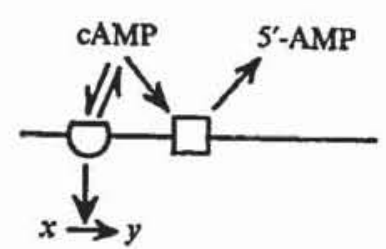

(3)

Figure 2. Hypothetical relations of cAMP receptors and cAMP phosphodiesterase at the cell surface. The function of the receptors is the coupling of a process at the inner side of the membrane, formulated as the production of $y$ from a precursor $x$, to the external cAMP signal. The external cAMP hydrolysed by the phosphodiesterase reappears in the extracellular space as $5^{\prime}$-cAMP. The results discussed in the text indicate cAMP binding sites not identical with the catalytic sites of the phosphodiesterase and therefore rule out possibility (1). Since the kinetics and specificity of cAMP binding and hydrolysis are different, possibility (2) is improbable. These results suggest independent interaction of both sites with free cAMP (possibility (3).

1974; Gerisch, Malchow \& Hess 1974). Their number in aggregating cells is about $5 \times 10^{5}$ per cell, their apparent dissociation constant in the order of $10^{-7} \mathrm{M}$ or less. These binding sites are distinguished from the phosphodiesterase by different kinetics and specificity, indicating independent interaction of both these sites with free cAMP (figure 2, right).

\section{Mechanism of chemotaxis}

Studies limited to the interaction of individual receptor molecules with cAMP cannot answer the question how an amoeboid cell recognizes a concentration gradient. The problem remains how the differential activation of receptors at different loci of the cell surface is translated into the directional formation of pseudopods. For the chemotaxis of $D$. discoideum cells the following statements can be made (figure 3, plate 3): (1) Cells migrate towards the source of cAMP more or less precisely in a straight line. They do not show random changes of direction that would correspond to the 'tumbling' of chemotactic bacteria (Macnab \& Koshland 1972;

\section{DesGription of plate 3}

Frgure 3. Capillary assay of the chemotactic response to cAMP. Aggregating cells respond to microcapillaries with a tip width of about $0.2 \mu \mathrm{m}$ filled with $10^{-4}$ to $10^{-5} \mathrm{M}$ cAMP. (In the particular case shown, the concentration was $10^{-6} \mathrm{M}$ and cAMP release was enhanced by iontophoresis (Robertson, Drage \& Colieu 1972). The strain used was a mutant (ga 93) forming large aggregation territories, which exhibits a particularly clear chemotactic response.) Photographs were taken at the following times: $a$, shortly before, $b$, immediately after, the capillary was introduced, $c 30, d 90, e 120, f 150 \mathrm{~s}, g 5, h 8 \mathrm{~min}$ later. $i$ shows the tracks of the cells, the circles indicating distances of 50 and $100 \mu \mathrm{m}$, respectively, around the capillary. The two cells labelled in (a) with an arrow demonstrate the formation of a new moving front in response to the gradient, with an intermediate T-shaped state ( $c$ to $f)$. 
Berg \& Brown 1972). (2) The pulsatile activity of aggregation centres is not necessary for chemotaxis. This follows from the chemotactic orientation towards centres of a mutant that releases the attractant continuously (Gerisch 1971), and also from the response to cAMP continuously diffusing out of microcapillaries. (3) Local stimulation of the cell surface induces the protrusion of pseudopods from the stimulated area. (4) Any part of the cell can function as the moving front, ruling out a fixed polarity of the cell as well as the restriction of cAMP

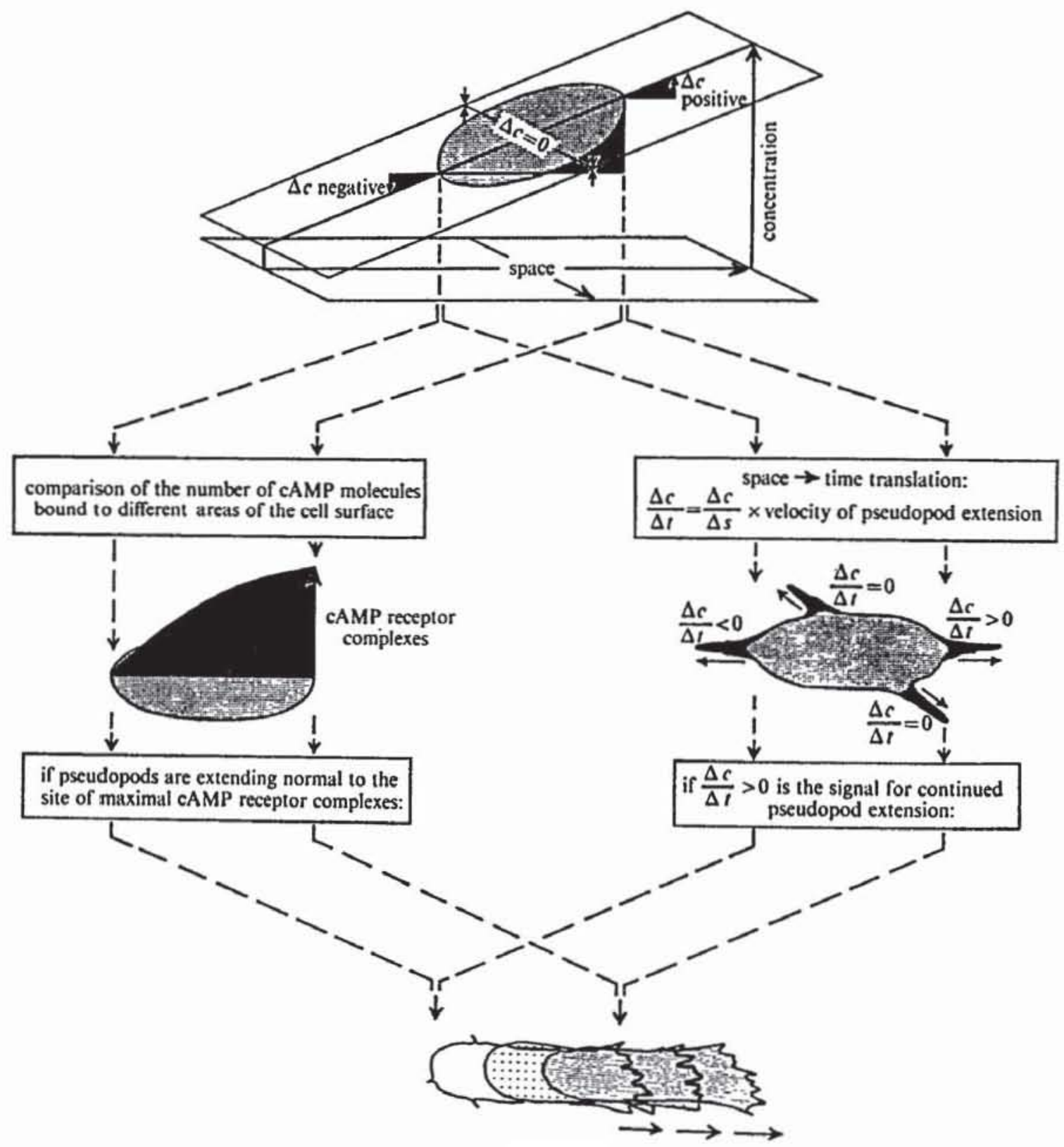

Frourg 4. Top: An amoeboid cell (shadowed area) can apply two principles for the detection of external concentration gradients: (1) the measurement of concentration differences along its surface, and (2) measuremotility. The external gradient is represented by extending pseudopods. Left: Principle (1) requires no cell complexes per cell surface area. It is suresented by the spatial distribution of the number of cAMP receptor of maximal cAMP binding. Right: Principle (2) continued pseudopod formation is stimulated at the area pseudopods which recognize the change for (2) implies that the spatial gradient is sensed by extending for continued pseudopods formation (bottom). 
receptors to circumscribed areas of the surface. (5) Moving fronts compete with each other, so that the induction of a new front tends to inactivate the previous one.

For the detection of spatial gradients, single cells can use either one of two principles (figure 4): (1) The direction of a gradient can be directly obtained by comparing the number of attractant molecules bound to different parts of the cell surface. (2) The cell can respond primarily to temporal changes of the attractant concentration that occur when either the whole cell or organelles change their position relative to a gradient. Since the whole cells do not move into alternating directions during their chemotactic response, the second principle is applicable to $D$. discoideum cells only in the version that pseudopods protruded into different directions act as sensors which, while expanding, are stimulated by temporal changes of the number of cAMP molecules bound to their surface (figure 4, right).
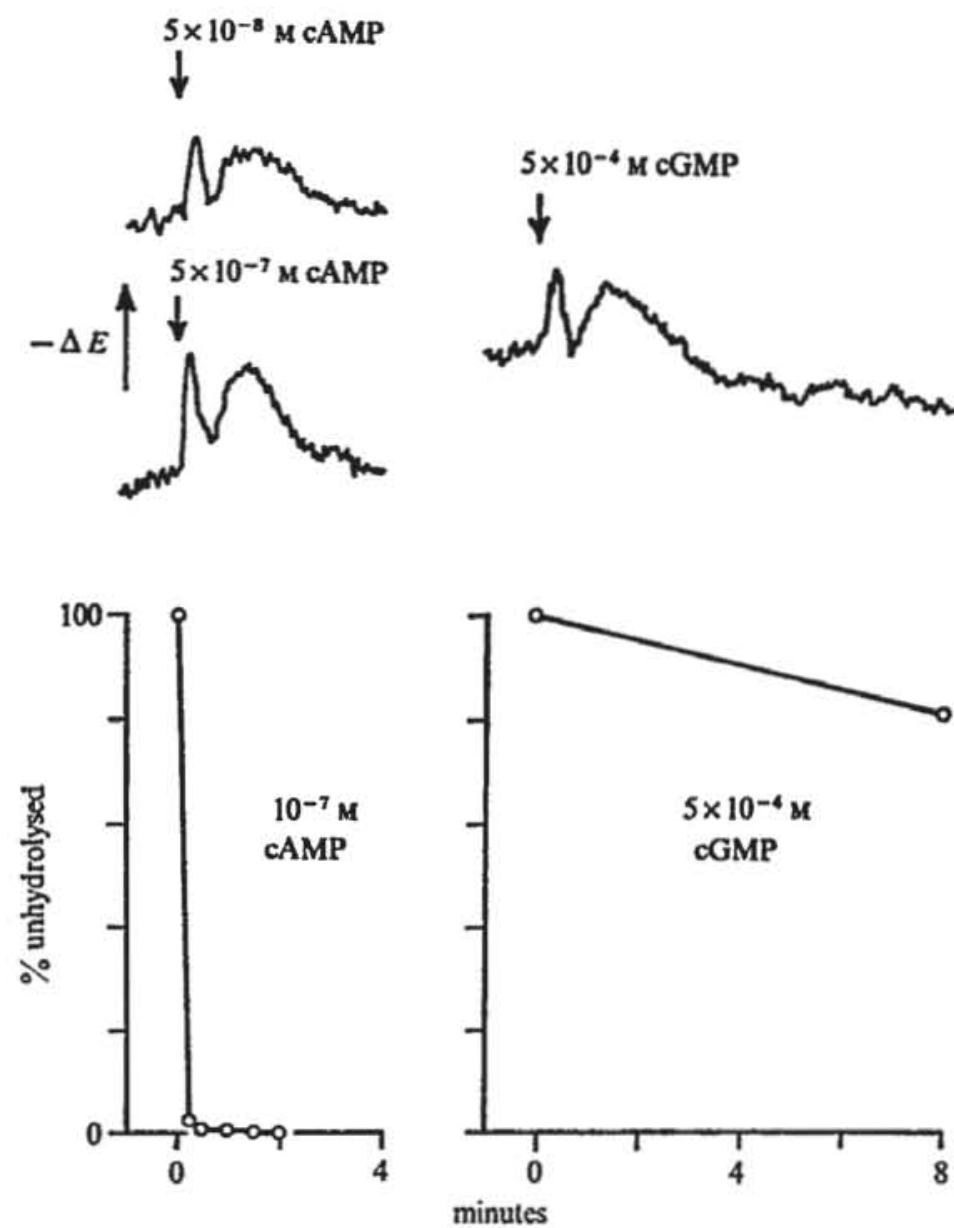

Figure 5. Gellular responses to concentration steps. Top: Responses to cAMP and other cyclic nucleotides can be recorded in cell suspensions using light scattering changes as an indicator. The time course of these responses is largely independent of the amplitude of the concentration change (left), and is unchanged if cAMP is replaced by a high concentration of cGMP (right). Cells have been used in the aggregationcompetent state, 8-9 h after end of growth. The strain was Ax-2. Bottom: Because of the cell-surface phosphodiesterase present, the lifetime of CAMP is short compared to the length of the responses (left). The long lifetime of cGMP (right) shows that the time course of the responses is not affected by the continued presence of the chemical signal. By adding $1 \times 10^{-8} \mathrm{M}$ [ $\left.{ }^{3} \mathrm{H}\right] \mathrm{CAMP}$ together with cGMP, it can be shown that cyclic nucleotide binding to the cell surface is equally extended under these conditions (Malchow \& Gerisch 1974). This indicates that the time pattern of the responses is not determined at the level of cAMP receptor interaction, but rather at a later step of signal processing. Conditions as in Malchow \& Gerisch (1974) and Gerisch \& Hess (1974). 
A distinction between principles (1) and (2) can be made if the type of signal input is known to which the chemoreceptor system responds. Cyclic AMP causes responses which can be easily recorded in cell suspensions since they are associated with light scattering changes (Gerisch \& Hess 1974; Gerisch et al. 1974). These responses are induced by a rapid increase of the cAMP concentration with time, and they invariantly decay with a constant time course. They do so, as expected, when the stimulating CAMP is rapidly hydrolysed by the cell surface phosphodiesterase. More important, the same response pattern is obtained with cGMP which, in accord with its weak chemotactic activity, has to be applied in high concentrations and hence has a much longer lifetime in the medium (figure 5). If simultaneously with cGMP, labelled cAMP is applied, it can be shown that the receptor sites continue to bind the cyclic nucleotide, but the responses nevertheless decay with the normal time course. These results indicate that $D$. discoideum cells function like sensory cells that exhibit a typical 'on'-response. Our results fit, therefore, to possibility (2) which says that the adequate stimulus for the chemoreceptor system is a temporal change of the cAMP concentration. These results, however, do not strictly rule out possibility (1), according to which the gradient detecting system would also recognize cAMP signals not changing with time.

\section{Signal relaying}

Chemotactic stimuli are propagated in cell layers as excitation waves apparently without decrement. This means that the signals are relayed by the chemotactically responding cells, indicating a signal output/input ratio $>1$. This means that any cell in an aggregation field can

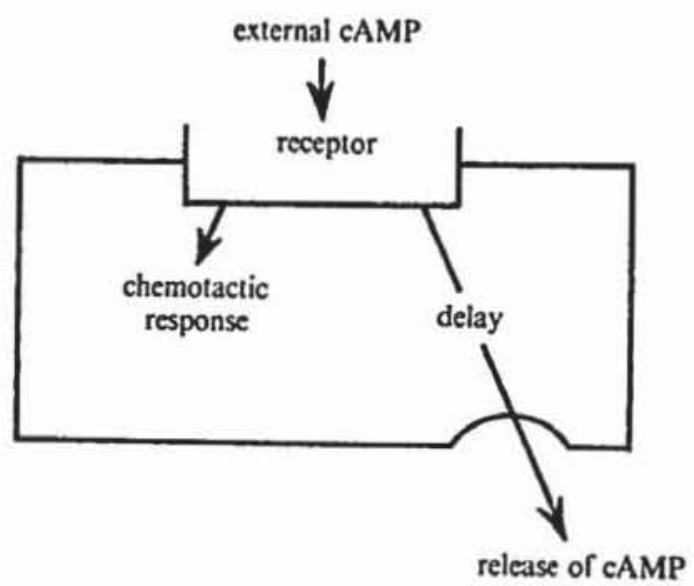

FIGURE 6. Proposed functional link of cAMP receptors both to the control of cell motility, necessary for orientated cell movement in cAMP gradients, and to the control of cAMP release, accounting for signal amplification in a relay system. It is not proven that both these functions are linked to the same receptor type.

function as a signal amplifier. The assumption that cAMP is not only a chemotactic agent but also the transmitter in the relay system (Robertson et al. 1972), implies that the cAMP-signals are processed by the receptors to target structures which control the external cAMP level (figure 6). To measure the output of cells in response to small cAMP-pulses, the extracellular cAMP levels in suspensions of aggregation-competent cells have been determined before, and in short intervals after stimulation by $5 \times 10^{-8} \mathrm{M}$ cAMP. Figure 7 shows that the cAMP level was modulated with an amplitude about tenfold higher than the signal amplitude. In cells of the particular stage used, the induced increase of the extracellular cAMP concentration was followed by a depression which in turn was followed by a second smaller maximum. 
Although experiments with a better time resolution are necessary to obtain the exact time course of the response, it appears likely from these initial data that the cAMP pulses triggered a damped oscillation of the extracellular cAMP level. The two sites which are possibly controlled by the cAMP activated receptors in the process of signal amplification, are adenylate cyclase (Rossomando \& Sussman 1973) and cAMP transport sites in the plasma membrane (figure 8).
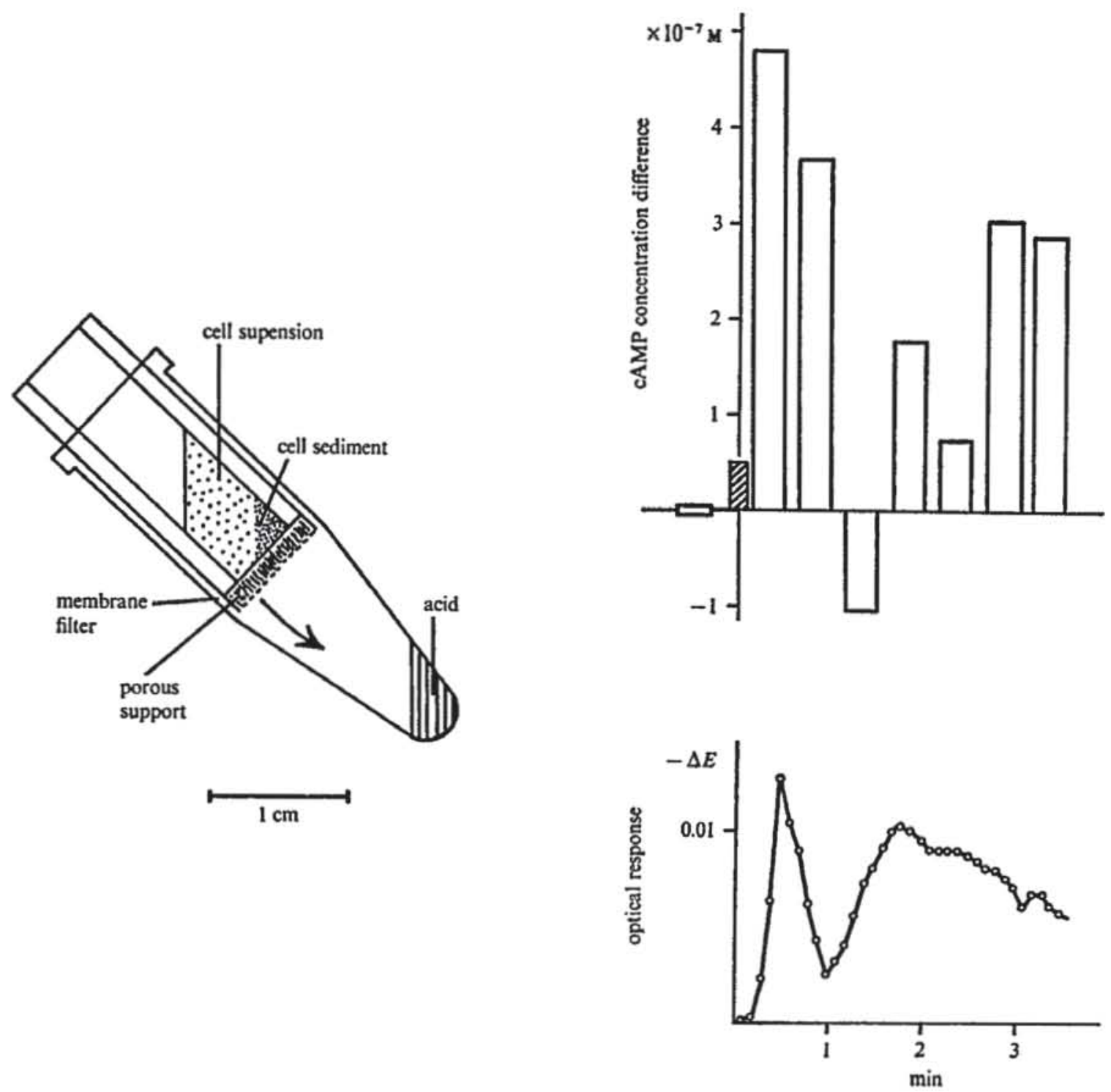

Figure 7. cAMP induced cAMP release. Left: Suspended cells were stimulated by a cAMP pulse of $5 \times 10^{-8} \mathrm{M}$. In intervals before and after stimulation the intercellular medium was rapidly separated from the cells by centrifugation through a $1.2 \mu \mathrm{m}$ pore size millipore filter, and any phosphodiesterase immediately inactivated by either perchloric or hydrochloric acid. cAMP was determined by the protein binding assay (Gilman I970). Top: Change of extracellular cAMP concentration as a function of time after stimulation. Means from 7 experiments. The 0 value of the ordinate represents the concentration before the cAMP pulse. The mean base level before stimulation was $5 \times 10^{-7} \mathrm{M}$ cAMP, the cell concentration $2 \times 10^{8} / \mathrm{ml}$, other conditions as in Gerisch \& Hess (1974). The cells were harvested in the aggregation-competent state $8 \mathrm{~h}$ after end of growth. The shaded column indicates the amplitude of the stimulating pulse. The strain was Ax-2. Bottom: Means of light scattering changes in the same experiments as on top. These changes are equivalent to those shown in figure 5. Spontaneous oscillations (figure 9 ) had been suppressed by cAMP pulses before the beginning of the experiment. All data from Wick (1974). 


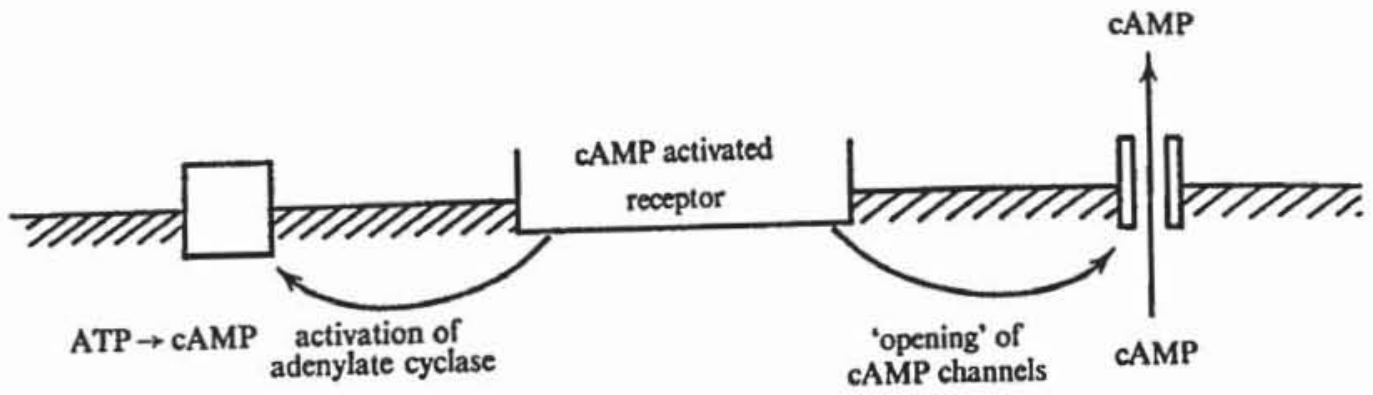

(1)

activated cAMP synthesis
(2) induced cAMP transport

Figure 8. Possible target structures for the functioning of cAMP receptors in signal relay.

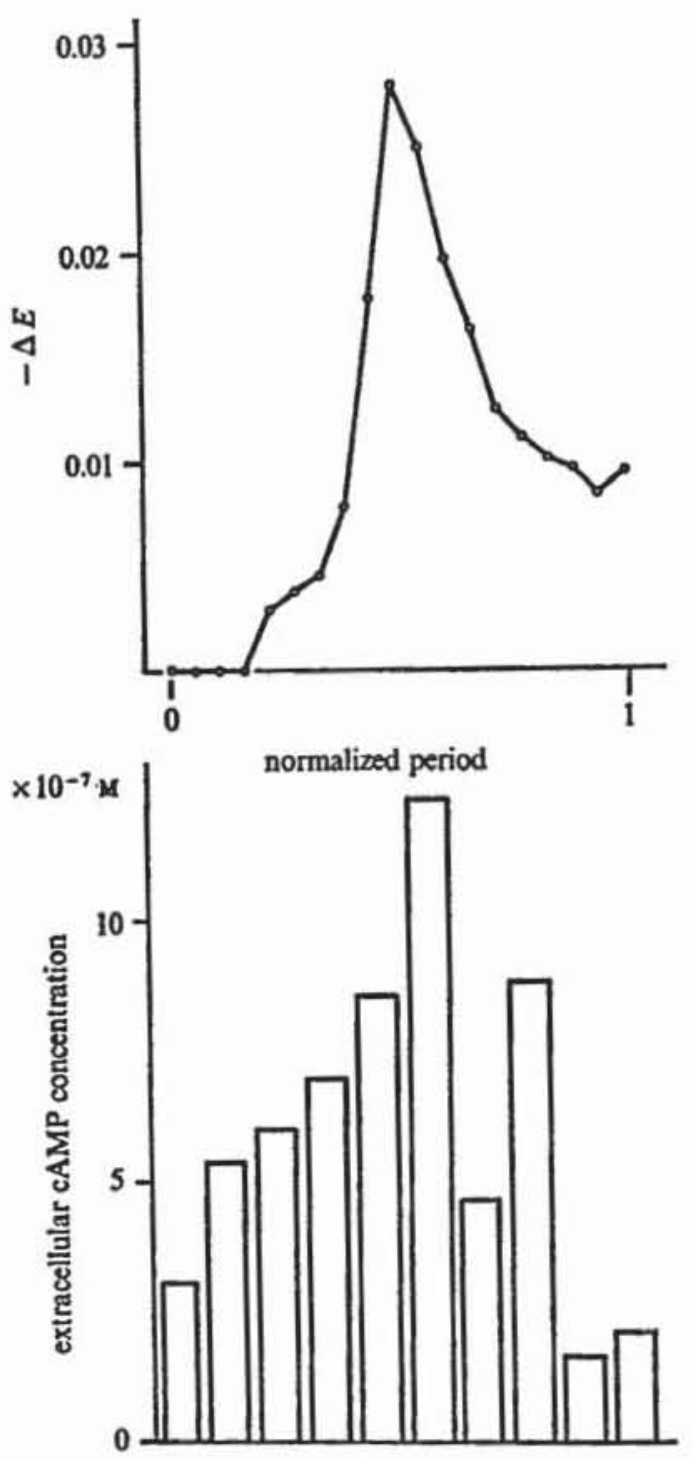

FigurE 9. Spontaneous periodicity of extracellular cAMP in cell suspensions. Conditions as in figure 7, except that no cAMP pulses were applied. Top: Light scattering changes during one period. Means from 4 experiments. Bottom: Variation of extracellular cAMP concentrations in the same experiments. The period lengths were normalized to 1, the real periods varied between 6 and $9 \mathrm{~min}$. In each experiment the peaks of light scattering were used for relating the cAMP data to the phases of a cycle. Data from Wick (1974). 


\section{Periodic pulse generation}

Chemotactic pulses are generated spontaneously and periodically in aggregation centres, and since excised centres are easily replaced by newly formed ones, there are reasons to believe that also outside the centres the cells have the ability to create periodic pulses. To study the function of the oscillator that controls pulse generation, dense cell suspensions have been aerated in an optical cuvette. Under these conditions, cells in the aggregation stage spontaneously generate spikes of decreased light scattering with a period of about $8 \mathrm{~min}$ (Gerisch et al. 1974; Gerisch \& Hess 1974). Later on, the pattern usually changes into sinusoidal oscillations. Spike formation is often correlated with oscillations in the cytochrome b spectrum, indicating periodic processes inside the cells (Gerisch \& Hess 1974; Hess, Boiteux, Busse \& Gerisch 1975). In the extracellular space, the cAMP concentration oscillates concomitantly with spike formation (figure 9).

These results fit to four different models (figure 10): (1) The oscillator is a completely intracellular machinery, and only the output, cAMP, appears in the extracellular space. There

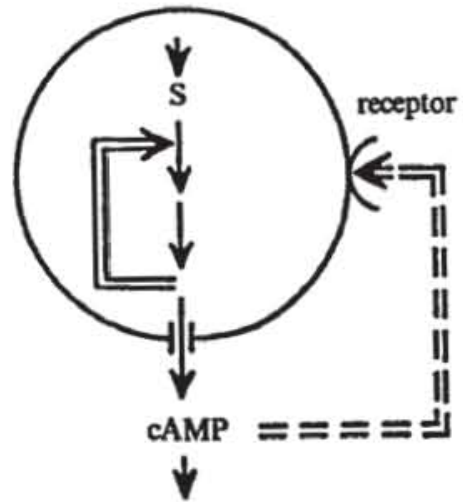

(1)
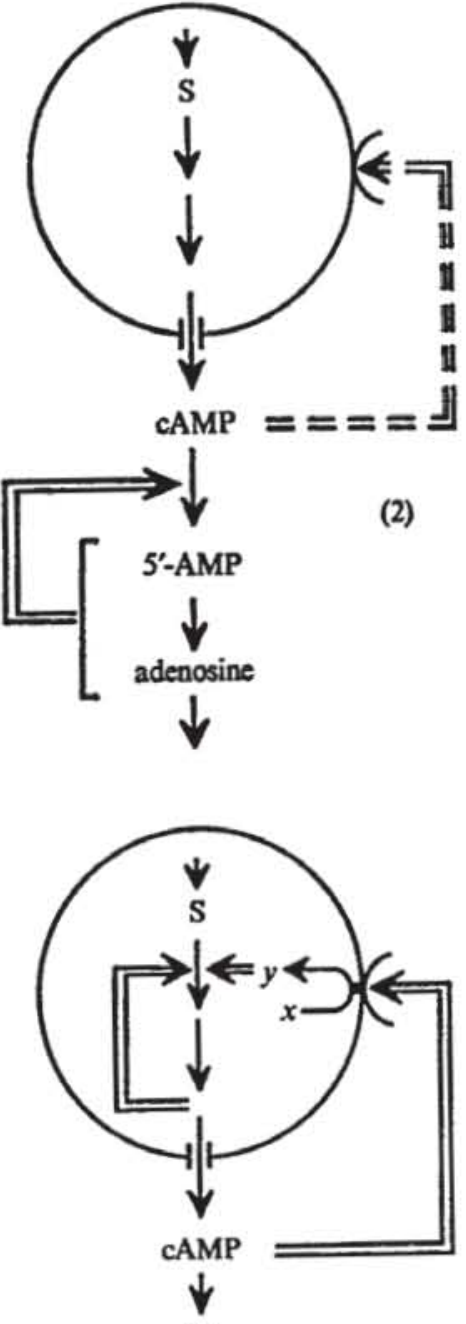

(4)

(3)

Figure 10. Simple network of biochemical cAMP oscillators. The circles separate intra- from extracellular processes. The double lines indicate control inputs; the broken lines those not connected to the oscillating system. 
is no connexion between the cAMP receptor at the cell surface and the oscillating system. (2) The oscillating system is totally outside the cell, and is constituted by cAMP and the products of phosphodiesterase and cell-surface phosphatase (Malchow \& Gerisch 1974). According to this model, the extracellular cAMP concentration would oscillate due to periodic regulation of the phosphodiesterase. The cell would play the two roles of a continuous cAMP source, and of an indicator of the external oscillations. (3) The oscillator has an intracellular and an extracellular part, both essential for its function. The extracellular part is formed by cAMP and its interaction with cell surface receptors. (4) The oscillator has an extracellular loop by which the oscillations can be modified; this loop, however, is not indispensable for periodic activity. Model (1) ignores the finding that cAMP applied extracellularly causes phase shifts of the oscillating system (Gerisch \& Hess 1974). Model (2) can be ruled out because 5'-AMP, which would be part of an extracellular cAMP oscillator, has no effect on the oscillations when externally applied. Both models (3) and (4) are in accord with our results, and we have only a weak argument in favour of model (4). In this model the properties of model (1) and (3) are combined. The argument is based on the existence of sinusoidal oscillations to which no spikes are coupled, and which are much less sensitive to extracellular cAMP than spike generation. Possibly these sinusoidal oscillations indicate the action of the oscillator in absence of an extracellular cAMP loop as indicated by model (1). Model (3) represents an excitable cell that would regulate its cAMP output in response to external signals, a condition that would be sufficient for signal relaying and also for the formation of spiral-shaped activity patterns in 'excitable media' (Winfree 1974).

Both in models (3) and (4) cAMP can act as an extracellular signal that modifies the oscillations via activation of cell surface receptors. These transform by an unknown mechanism the extracellular signal into an intracellular one ( $y$ in figure 10) which is connected to or is part of the oscillating system, and possibly but not necessarily is also cAMP. Of course, extracellular cAMP does not only feed back to the particular cell which produced it, but also interacts with other cells, in this way synchronizing the activities of individual cells in suspension.

For this functioning of cAMP as a coupling factor for periodic cellular activities, and also for its role in signal relaying, it is essential that the extracellular cAMP concentration changes periodically. In this context it is important that a non-linear kinetics enables the cell-surface phosphodiesterase to reduce, after a pulse, the cAMP concentration rapidly down to the noise level of the receptor system (Malchow, Tuchila \& Nanjundiah 1975). The combination of receptors and hydrolysing enzyme in a system that transfers periodic signals by a diffusible transmitter through an intercellular space, makes signal transmission in Dictyostelium comparable to synaptic transmission by acetylcholine. The expanded time scale of the periods in Dictyostelium is certainly an advantage for the experimental analysis of both a receptor system and a biochemical oscillator (Goldbeter 1975 ) functioning in morphogenetic signal transmission.

\section{REFERENCEs (Gerisch et al.)}

Berg, H. C. \& Brown, D. A. 1972 Nalure, Lond. 239, 500.

Bonner, J. T., Barkley, D. S., Hall, E. M., Konijn, T. M., Mason, J. W., O'Keefe, G. \& Wolfe, P. B. 1969 Devl. Biol. 20, 72.

Cohen, M. H. \& Robertson, A. I971 J. theor. Biol. 31, 119.

Durston, A. 1974 Devl. Biol. 37, 225.

Gerisch, G. 1968 Curr. Top. Develop. Biol. 3, 157.

Gerisch, G. 1971 Naturwissenschaften 58, 430. 
Gerisch, G. \& Hess, B. 1974 Proc. natn. Acad. Sci. U.S.A. 71, 2118. Gerisch, G., Malchow, D. \& Hess, B. 1974 In Biochemistry of sensory functions. Mosbacher Kolloquium 1974
(ed. L. Jaenicke). Heidelberg: Springer-Verlag.

Gerisch, G., Normann, I. \& Beug, H. 1966 Naturwissenschaften 53, 618.

Gilman, A. G. 1970 Proc. natn. Acad. Sci. U.S.A. 67, 305.

Goldbeter, A. 1975 Nature, Lond. 253, 540. Hess, B., Boiteux, A., Busse, H. G. \& Gerisch, G. 1975 In Membranes, dissipative structures and function (ed. G.
Nicolis). New York: Wiley.

Konijn, T. M. 1972 Adv. cyclic Nucleotide Res. 1, 17.

Macnab, R. M. \& Koshland, D. E. Jr 1972 Proc. natn. Acad. Sci. U.S.A. 69, 2509.

Malchow, D., Fuchila, J. \& Jastorff, B. 1973 F.E.B.S. Lett. 34, 5.

Malchow, D. \& Gerisch, G. 1973 Biochem. biophys. Res. Commun. 55, 200.

Malchow, D. \& Gerisch, G. 1974 Proc. natn. Acad. Sci. U.S.A. 71, 2423.

Malchow, D., Nägele, B., Schwarz, H. \& Gerisch, G. 1972 Eur. J. Biochem. 28, 136.

Malchow, D., Tuchila, J. \& Nanjundiah, V. 1975 Biochem. biophys. Acta 385, 421.

Pannbacker, R. G. \& Bravard, L. J. 1972 Science, N.Y. 175, 1014.

Robertson, A., Drage, D. J. \& Cohen, M. H. 1972 Science, N.Y. 175, 333.

Rossomando, E. F. \& Sussman, M. 1973 Proc. natn. Acad. Sci. U.S.A. 70, 1254.

Shaffer, B. M. 1962 Adv. Morphogen. 2, 109.

Wick, U. 1974 Diplomarbeit, Universität Tübingen.

Winfree, A. T. 1974 SIAM-AMS Proc. 8.

\section{Discussion}

Sir ANDRew Huxley, F.R.S. (Department of Physiology, University College London, Gower Street, London WC1E 6BT)

One of Gerisch's slides showed changes of cyclic AMP concentration in a suspension of his amoebae after addition of a trigger dose of cyclic AMP. The initial concentration was $5 \times 10^{-7} \mathrm{M}$, and the increase due to the added material was $5 \times 10^{-8} \mathrm{M}$. Does this mean that the response was triggered by a $10 \%$ change in the concentration to which each cell was exposed, or were there perhaps much larger concentration changes locally at the place where the dose was applied?

\section{G. Gerisch}

We injected $1 \mu \mathrm{l}$ of $10^{-4} \mathrm{M}$ cyclic AMP into $2 \mathrm{ml}$ of a cell suspension which is being rapidly mixed. Since cyclic-AMP binding to the receptors is fast, the injection and mixing techniques used do not strictly rule out that, for a short time, a small portion of the cells are stimulated by a high concentration step, and that these cells catalyse the stimulation of others. In various respects, our techniques have to be improved to answer the question. This reservation does not touch the point I wanted to make, namely that the signals we feed in are amplified by the cells.

\section{P. M. Newsome (Beecham Pharmaceuticals Research Division, Chemotherapeutic Research Centre, Brockham Park, Betchworth, Surrey RH3 7AJ)}

I was fascinated by Gerisch's account of cellular communication in the slime mould. Of course cellular aggregation requiring cell-to-cell communication is important in many other situations. One which springs to mind is the accumulation of macrophages and polymorphonuclear leucocytes at inflammatory foci in higher organisms. I wonder whether Dr Gerisch might comment on possible significance of periodic cell-to-cell signalling in other situations.

\section{G. Gerisch}

The nervous system and the vertebrate heart are of course the best examples. Periodic clectrical potential changes have been observed in the unicellular green alga, Acetabularia 
mediterranea (B. Novak \& F. W. Bentrup, Planta (Berlin) 108, 227 (1972)). Periodicity of signalling has been suggested to be a basis of pattern formation in developmental systems (G. Gerisch in Current topics in developmental biology (A. Monroy \& A. A. Moscona, Eds.), Vol. 3, p. 95, New York: Academic Press (1968). B. C. Goodwin \& M. H. Cohen, J. theoret. Biol. 25, 49 (1969)). A specific example, the determination of the number of somites, has been recently discussed by J. Cooke, Proceedings of the 1975 ICN-UCLA Symposium on Developme tal Biology (D. McMahon \& C. F. Fox, Eds.), W. A. Benjamin Inc. 